\title{
Leaf Position Prevails Over Plant Age and Leaf Age in Reflecting Resistance to Late Blight in Potato
}

\author{
M. H. P. W. Visker, L. C. P. Keizer, D. J. Budding, L. C. Van Loon, L. T. Colon, and P. C. Struik
}

First, second, third, and fifth authors: Plant Research International, P.O. Box 16, 6700 AA Wageningen, the Netherlands; first and sixth authors: Crop and Weed Ecology, Department of Plant Sciences, Wageningen University, P.O. Box 430, 6700 AK Wageningen, the Netherlands; and fourth author: Section of Phytopathology, Faculty of Biology, Utrecht University, P.O. Box 800.84, 3508 TB Utrecht, the Netherlands.

Accepted for publication 27 January 2003.

\begin{abstract}
Visker, M. H. P. W., Keizer, L. C. P., Budding, D. J., Van Loon, L. C., Colon, L. T., and Struik, P. C. 2003. Leaf position prevails over plant age and leaf age in reflecting resistance to late blight in potato. Phytopathology 93:666-674.

The effects of plant age, leaf age, and leaf position on race-nonspecific resistance against Phytophthora infestans were investigated in a series of field and controlled environment experiments with five different potato (Solanum tuberosum) cultivars. Leaf position proved to be the most sig-

nificant factor; apical leaves were far more resistant to late blight than basal leaves. Plant age and leaf age had only minor effects; therefore, the resistance of a specific leaf remained about the same during its entire lifetime. The gradual increase in late blight resistance from basal leaves to apical leaves appeared to be a general effect, irrespective of cultivar, growing conditions, or resistance test. Therefore, it is important to consider leaf position in tests for late blight resistance, because contrasts in resistance may be ascribed erroneously to differences between genotypes or treatments, whereas they are actually caused by differences in leaf position.
\end{abstract}

Phytophthora infestans causes late blight, a serious threat to potato (Solanum tuberosum) production throughout the world. At present, late blight epidemics can be controlled only by frequent applications of fungicides, which are expensive, have practical limitations, and cause environmental concerns. A disease management strategy with high potential is the use of cultivars with durable resistance to late blight (10), and various approaches in breeding have been applied to accomplish this resistance (26). One method to obtain late blight-resistant potato cultivars is the introgression of major resistance $(R)$ genes. However, this approach is frustrated by the rapid development of new races of the pathogen, because compatible races of $P$. infestans have long been present for (combinations of) all $11 R$ genes that have been identified (36). Therefore, breeding for late blight resistance cannot rely on these $R$ genes alone, and should be directed toward race-nonspecific resistance (36), which appears to be more durable (14). Introgression of race-nonspecific resistance against $P$. infestans into modern potato cultivars is hindered by the uncertainty regarding the number of genes involved (12), the resistance mechanisms these genes trigger (similar to those controlled by the 11 known $R$ genes?) (34), and the durability of their effects (9). In addition, race-nonspecific resistance to late blight in potato has an unfortunate association with late foliage maturity (31): it appears nearly impossible to breed the desired, early-maturing cultivars with high levels of resistance (30).

The question what makes early-maturing cultivars different from late maturing ones regarding their late blight resistance has been addressed by studying the effects of factors that are most likely related to foliage maturity type, such as plant age and leaf position. Several studies have been published about the changes in resistance against $P$. infestans with increasing plant age in potato $(5,8,11,13,20,27,29)$. Results of these experiments are difficult to

Corresponding author: M. H. P. W. Visker; E-mail address: Marleen.Visker@wur.nl

Publication no. P-2003-0331-01R

(c) 2003 The American Phytopathological Society compare, because different methods have been used for cultivation and evaluation of the plants and large variations were found within some experiments. Still, these studies permit some general conclusions: very young plants are susceptible to late blight, plants of intermediate age are the most resistant, and old plants become more susceptible again $(20,27)$. However, the increase in susceptibility at higher plant ages also may depend on the general level of late blight resistance of the genotype, because Carnegie and Colhoun (5) found that susceptible cultivars appeared to become more susceptible at higher plant ages, whereas the more resistant cultivars appeared to become even more resistant. In addition to the changes in resistance against $P$. infestans with increasing plant age, different leaves of the same potato plant also vary in their level of resistance, irrespective of plant age. Research on the effects of leaf age and leaf position has been scarce, but apical leaves appear to be more resistant to late blight than basal leaves of the same plant at the same time $(4,5,16,19,20)$.

The objective of this study was to investigate the effects of plant age, leaf age, and leaf position on the linear growth rate of lesions of $P$. infestans. Plant age, leaf age, and leaf position were chosen as simple and objective parameters expected to be correlated with foliage maturity type. The linear lesion growth rate of $P$. infestans was the metric value representing late blight resistance (6). The experiments were designed to allow conclusions about the interaction between changes in resistance to late blight with increasing plant age and the variation in resistance within a plant (leaf age and leaf position).

\section{MATERIALS AND METHODS}

Two small-scale experiments were performed first and the results of these experiments were used to optimize the design of the subsequent experiments. Most practical details were similar in all five experiments. Differences in plant material (in vitro plantlets or seed tubers), growing conditions of the plants (climate-controlled or field), and tests for late blight resistance (leaves detached or not) were introduced to test the validity of the results. 
Plant material and planting. The experiments were performed with five potato (Solanum tuberosum L.) cultivars: Eersteling, Spunta, Alpha, Pimpernel, and Robijn. These cultivars represent the current northwestern-European variation for resistance to late blight and for foliage maturity type, as indicated by the Dutch list of varieties of field crops (1-3) (Table 1). None of these cultivars contain any of the 11 known $R$ genes for resistance against $P$. infestans (W. G. Flier, personal communication).

In vitro-propagated plantlets were used in experiments 1, 3, 4, and 5 as planting material to avoid effects of differences in physiological age of seed tubers on resistance to late blight (28). In vitro plantlets were maintained on Murashige and Skoog (MS) medium (21) supplemented with sucrose $\left(15 \mathrm{~g} \mathrm{liter}^{-1}\right)$ and mannitol $\left(15 \mathrm{~g} \mathrm{liter}^{-1}\right)$ at $21^{\circ} \mathrm{C}$ with a photoperiod of $16 \mathrm{~h}$ of fluorescent light (Philips TLD 50W/84oHF, two lamps per square meter at $0.25 \mathrm{~m}$ above the plants). Two weeks before planting, fresh cuttings of the in vitro plantlets were transferred onto new MS medium supplemented with sucrose $\left(30 \mathrm{~g} \mathrm{liter}^{-1}\right)$ to induce rooting and grown at the above described temperature and light conditions. At planting, each plantlet was transplanted into a black 5-liter pot filled with potting soil and covered with a small transparent container to prevent transplant shock; containers were removed after 1 or 2 days. Growing plants were pruned by removing axillary buds to have only one main stem, and water was supplied when needed to avoid premature senescence due to drought stress.

Plant age, leaf age, and leaf position. Plant ages were created in experiments 1,3 , and 4 by planting with intervals prior to inoculation, and intentionally not by simultaneous planting, followed by a series of late blight resistance tests at different times (8). The goal of planting at intervals was to minimize the number of resistance tests, because each test requires fresh inoculum. The quality of the inoculum cannot be controlled (22) and this inevitable variation easily can result in variation in the outcome of the resistance tests. Using a series of late blight resistance tests at different times after simultaneous planting would have resulted in intertwining the effects of plant ages and resistance tests. Plant ages were determined on the days the tests for late blight resistance were initiated and counting started from the days the in vitro plantlets had been transplanted into the pots with soil. Plant ages ranged from 23 to 66 days old (after planting).

Assignment of leaf ages in experiments 1,3, and 4 was enabled by marking all newly appeared compound leaves that had reached a total length of at least $3 \mathrm{~cm}$ with a date-tag twice a week during plant growth. Leaf ages were determined on the days the tests for late blight resistance were initiated and counting started from the days the leaves had been tagged. Because leaves were tagged only twice a week, several leaves of the same plant could be assigned to the same leaf age; therefore, leaf ages actually were leaf age classes, but will be referred to as leaf ages hereafter. Leaf ages ranged from 2 to 53 days old (after reaching a length of $3 \mathrm{~cm}$ ).

Leaf positions were assigned in experiments $1,3,4$, and 5 on the days the tests for late blight resistance were initiated and were counted from base to apex. Leaf positions ranged from 1 (base) to
19 (apex). Fully decayed leaves were included for assigning leaf positions but were not tested for resistance.

Leaves of experiments 1, 3, and 4 were selected prior to inoculation with $P$. infestans to restrict the total number of leaves to be tested to fit the maximum capacity of the testing facility. Selection was based on leaf age and resulted in random sampling for leaf position. All available leaf ages on each single plant were included in the late blight resistance tests. If a plant had two leaves of the same age (class), the lower one was tested. If a plant had three leaves of the same age (class), the lower and the upper ones were tested (and treated as independent observations). Individual plants never had more than three leaves of the same age (class). This selection resulted in testing a total of one or two leaves for the youngest plants (23 days old) and up to 12 leaves for the oldest plants (66 days old).

Testing for resistance to late blight. The late blight resistance tests for experiments 1, 2, 3, and 4 were adopted from Vleeshouwers et al. (35), because this laboratory test ensured controlled test conditions, was practically feasible for this study, and has proven to be a good alternative for field testing. Seven tests for late blight resistance were performed: one for each of the experiments 1, 2, and 4, and four for experiment 3. Performing multiple tests was necessary, because each individual test required the maximum capacity of the testing facility.

Leaves were cut from the plants on the day of inoculation, placed with the abaxial surface upward on wet filter paper in trays of 45 by 30 by $8.5 \mathrm{~cm}$, and inoculated with a complex isolate of $P$. infestans (Mont.) de Bary (IPO82001; race 1.2.3.4.5.6.7.10.11) (9). Each of five leaflets per compound leaf was inoculated with a $10-\mu$ droplet of $P$. infestans suspension containing $5 \times 10^{4}$ zoospores per $\mathrm{ml}$. After inoculation, every tray was wrapped in a transparent plastic bag to ensure maximum relative humidity (RH). Inoculated leaves were incubated in a growth chamber with a photoperiod of $16 \mathrm{~h}$ of fluorescent light (Philips TLD 36W/84o, four lamps per square meter at $0.35 \mathrm{~m}$ above the leaves) at day and night temperatures of 18 and $15^{\circ} \mathrm{C}$, respectively (35).

Over $99 \%$ of all inoculations resulted in growing lesions and only these successful inoculations were examined further. Each lesion that was caused by late blight infection was measured three times during the phase of linear growth (7), 3, 4, and 5 days after inoculation. The largest length and width (perpendicular to the length) of each lesion were measured, and the ellipse area $(A=$ $1 / 4 \times \pi \times$ length $\times$ width) was calculated. This ellipse area was used to calculate the mean half diameter ( $\approx$ radius) of the lesion $(r=\operatorname{sqrt}[A / \pi])$, which was used to estimate the rate of linear lesion growth of $P$. infestans by linear regression over the three successive days of repeated measurements of the same lesion (35). The linear lesion growth rates were used as input for the data analyses. Compound leaves were the smallest experimental units, consisting of five leaflets each.

Data analysis. The linear lesion growth rates of $P$. infestans were analyzed with the residual maximum likelihood (REML) method (23) of GenStat 5 (VSN International Ltd., Oxford), be-

TABLE 1. Average linear lesion growth rates of Phytophthora infestans for the five potato cultivars that were used in the various experiments

\begin{tabular}{|c|c|c|c|c|c|c|c|}
\hline \multirow[b]{2}{*}{ Cultivar } & \multirow[b]{2}{*}{ Foliage maturity type ${ }^{w}$} & \multirow[b]{2}{*}{ Late blight resistance $\mathrm{e}^{\mathrm{x}}$} & \multicolumn{5}{|c|}{ Linear lesion growth rate $\left(\mathrm{mm} \mathrm{day}^{-1}\right)^{\mathrm{y}}$} \\
\hline & & & Experiment 1 & Experiment 2 & Experiment 3 & Experiment 4 & Experiment 5 \\
\hline Eersteling & 8.5 & 2 & $\ldots$ & $5.68 \mathrm{a}$ & $5.24 \mathrm{a}$ & $5.41 \mathrm{a}$ & $4.36 \mathrm{a}$ \\
\hline Spunta & 7 & 5 & $\ldots$ & $\ldots$ & $4.37 \mathrm{~b}$ & $4.55 \mathrm{~b}$ & $2.34 \mathrm{c}$ \\
\hline Alpha & 4 & 5 & 4.77 & $\ldots$ & $4.55 \mathrm{~b}$ & $4.50 \mathrm{~b}$ & $3.64 \mathrm{~b}$ \\
\hline Pimpernel & 3.5 & 8 & $\ldots$ & $3.62 \mathrm{~b}$ & $\ldots$ & $\ldots$ & $\ldots$ \\
\hline Robijn & 3 & 8 & $\ldots$ & & $3.79 \mathrm{c}$ & $3.79 \mathrm{c}$ & $2.46 \mathrm{c}$ \\
\hline $\operatorname{LSD}_{0.05^{\mathrm{z}}}$ & $\ldots$ & $\ldots$ & $\ldots$ & 0.30 & 0.30 & 0.42 & 0.38 \\
\hline
\end{tabular}

${ }^{\mathrm{w}}$ Data of long-term field evaluations for foliage maturity type (1 = late, $9=$ early), obtained from the Dutch list of varieties of field crops (1-3).

${ }^{x}$ Data of long-term field evaluations for resistance to late blight $(1=$ susceptible, $9=$ resistant $)$, obtained from the Dutch list of varieties of field crops $(1-3)$.

y Different letters within a column indicate significant differences.

${ }^{\mathrm{z}} \mathrm{LSD}=$ least significant difference. 
cause the studied biological system implied highly unbalanced experiments (several combinations of factors could not be realized; e.g., old leaves on young plants). To avoid weak statements due to this unbalance, if less than three growing lesions were present for a factor or combination of factors, these data were removed beforehand. The REML method always applies fixed and random factors: fixed factors are the ones of interest (e.g., plant age), and random factors the ones inherent to the experimental design (e.g., block). Several statistical analyses were performed to determine which factors were most informative (see Results; leaf position was preferred to leaf age for further analyses). Finally, averages and variances for the linear lesion growth rates of $P$. infestans were obtained with the relevant factors cultivar, plant age, leaf position, and all their interactions in the fixed part of the statistical analyses (REML), and the factors block, subblock, plot, and leaf in the random part of the statistical analyses. Relevant factors are indicated below in the experimental design of each experiment. The significance ( $P$ values) and the effects of the fixed part of the statistical analyses were obtained from the Wald statistics $\left(\chi^{2}\right.$ distributed) as produced by the REML method. The REML method also produced least significant difference values for pairwise comparisons ( $t$ test), for which the variation between the five leaflets of a compound leaf was averaged, and used to estimate the variation between the replications within each experiment.

Experimental design: experiment 1. Potted in vitro plantlets of potato cv. Alpha were grown in a growth chamber (plant spacing $0.35 \mathrm{~m}$ ) at a photoperiod of $14 \mathrm{~h}$, day and night temperatures of 19 and $11^{\circ} \mathrm{C}$, respectively, and $\mathrm{RH}$ ranging between 65 and $75 \%$. Illumination was provided by Philips HPI-T 400W and Philips SON-T 400W lamps that were alternated at a density of three per square meter at $1 \mathrm{~m}$ above the pots. Leaves of all plants of this experiment were tested simultaneously for late blight resistance by cutting them off and inoculating them on the same day. The resistance test started 23 days after the last of seven weekly plantings; therefore, plants were $23,30,37,44,51,58$, or 65 days old (after planting) at the initiation of the test. As many as 13 different leaf positions (base to apex) were distinguished, representing 12 different leaf ages: 2, 6, 9, 13, 16, 21, 22, 28, 30, 35 , 37, and 43 days old (after reaching a length of $3 \mathrm{~cm}$ ). The experiment comprised 10 replications: the growth chamber was divided into 10 blocks, each containing seven plots that were randomly assigned to one of the seven plant ages. For the late blight resistance test, all leaves of all plants within one block (different plant ages) were randomized and distributed over a series of trays containing four or five leaves each. The statistical analysis (REML) comprised the factors plant age, leaf position, and their interaction as fixed, and the factors block, plot, and leaf as random.

Experimental design: experiment 2. Plants of potato cvs. Eersteling and Pimpernel were grown from commercially obtained seed tubers in the field (Wageningen, the Netherlands; plant spacing 0.35 by $0.7 \mathrm{~m}$ ) in the 1998 growing season. Tubers were planted on 29 April and leaves of all plants were tested simultaneously for late blight resistance by cutting them off and inoculating them on 18 June, when plants were $\approx 40$ days old (after emergence). In contrast to the other experiments, no exact leaf positions were determined, but five different positions were distinguished that were distributed evenly from base to apex. Therefore, leaf positions of this experiment were numbered I to V (base to apex), indicating their relative positions. Leaf ages were not determined. The experiment comprised 10 replications: the field was divided into 10 blocks, each containing two plots that were randomly assigned to one of the two cultivars. For the late blight resistance test, all leaves of all plants within one block (different cultivars) were randomized and distributed over a series of trays containing four or five leaves each. The statistical analysis (REML) comprised the factors cultivar, leaf position, and their interaction as fixed, and the factors block, plot, and leaf as random.
Experimental design: experiment 3. Potted in vitro plantlets of potato cvs. Eersteling, Spunta, Alpha, and Robijn were grown in growth chambers (plant spacing $0.35 \mathrm{~m}$ ) at a photoperiod of $16 \mathrm{~h}$, day and night temperatures of 19 and $11^{\circ} \mathrm{C}$, respectively, and RH ranging between 65 and $75 \%$. Illumination was identical to experiment 1 . Experiment 3 was conducted consecutively in four similar growth chambers; leaves of all plants that were grown in one growth chamber were tested simultaneously for late blight resistance by cutting them off and inoculating them on the same day. Resistance tests started 24 days after the last of seven weekly plantings; therefore, plants were $24,31,38,45,52,59$, or 66 days old (after planting) at the initiation of the tests. As many as 19 different leaf positions (base to apex) were distinguished, representing 15 different leaf ages: 4, 8, 11, 15, 18, 22, 25, 29, 32, 36, $39,43,46,50$, or 53 days old (after reaching a length of $3 \mathrm{~cm}$ ). The experiment comprised eight replications, distributed over four similar growth chambers. Each growth chamber was divided into two blocks (replications), which consisted of four subblocks each that were randomly assigned to one of the four cultivars. Every subblock contained seven plots that were randomly assigned to one of the seven plant ages. The total experiment comprised 224 plants: four growth chambers $\times$ two replications (blocks) $\times$ four cultivars (subblocks) $\times$ seven plant ages (plots). For the late blight resistance tests, all leaves of all plants within one subblock (same cultivar, different plant ages) were randomized and distributed over a series of trays containing four or five leaves each. The statistical analysis (REML) for each of the four growth chambers comprised the factors cultivar, plant age, leaf position, and all their interactions as fixed, and the factors block, subblock, plot, and leaf as random. An overall analysis of the entire experiment 3 was allowed, because similar effects with comparable residual mean squares (experimental errors) were found for all four tests for resistance. The factor growth chamber was added as a random factor to the overall statistical analysis (REML) of this experiment.

Several curves were fitted (GenStat 5) to describe the effects of leaf position on the linear lesion growth rate of $P$. infestans (data not shown). Logistic curves accounted for the highest percentage of variance (data not shown); therefore, such curves were fitted for each cultivar on the combined data of all plant ages and all leaf positions. Separate curves were fitted for each of the four growth chambers to enable statistical testing for parameter differences between cultivars with REML (GenStat 5). Curves were fitted for experiment 3 , because it was the most extensive set of data and, thus, ensured the most reliable fit and enabled statistical testing of the parameters.

Experimental design: experiment 4. Potted in vitro plantlets of potato cvs. Eersteling, Spunta, Alpha, and Robijn were grown in the field (Wageningen, the Netherlands; plant spacing $0.7 \mathrm{~m}$ ) in the 1999 growing season. The first plantlets were transplanted on 4 May, and subsequent plantings were on 25 May and 15 June. Pots first were kept inside, and then transferred to the field 2 or 3 days after planting, where the pots were dug into hills. Leaves of all plants of this experiment were tested simultaneously for late blight resistance by cutting them off and inoculating them on 9 July. The resistance test started 24 days after the last of three plantings with an interval of 21 days; therefore, plants were 24 , 45 , or 66 days old (after planting) at the initiation of the test. As many as 19 different leaf positions (base to apex) were distinguished, representing 13 different leaf ages: 4, 8, 11, 15, 18, 22, $25,29,32,36,39,43$, or 46 days old (after reaching a length of $3 \mathrm{~cm}$ ). The experiment comprised six replications: the field was divided into six blocks (replications), which consisted of four subblocks each that were randomly assigned to one of the four cultivars. Every subblock contained three plots that were randomly assigned to one of the three plant ages. This experiment consisted of 72 plants: six replications (blocks) $\times$ four cultivars (subblocks) $\times$ three plant ages (plots). For the late blight resistance test, all leaves of all plants within one subblock (same cultivar, different plant 
ages) were randomized and distributed over a series of trays containing four or five leaves each. The statistical analysis (REML) comprised the factors cultivar, plant age, leaf position, and all their interactions as fixed, and the factors block, subblock, plot, and leaf as random.

Experimental design: experiment 5. Potted in vitro plantlets of potato cvs. Eersteling, Spunta, Alpha, and Robijn were grown in a climate-controlled greenhouse (Wageningen, the Netherlands; plant spacing $0.35 \mathrm{~m}$ ) in the year 2002 at a photoperiod of $16 \mathrm{~h}$, day and night temperatures of 20 and $15^{\circ} \mathrm{C}$, respectively, and $\mathrm{RH}$ ranging between 70 and $80 \%$. Additional illumination was provided by Philips SON-T 400W lamps at a density of one per $2 \mathrm{~m}^{2}$ at $1 \mathrm{~m}$ above the pots. Plants were planted in the greenhouse on 28 March and leaves of all plants were tested simultaneously for late blight resistance by inoculating them on 2 May, when plants were 35 days old (after planting). As many as 16 different leaf positions (base to apex) were distinguished; leaf ages were not determined. In contrast to all previous experiments, leaves were not cut off from the plants; instead, resistance testing was performed on nondetached leaves on intact plants. Leaves were inoculated on the adaxial surface and incubation took place in the same greenhouse in which the plants were grown, with $\mathrm{RH}$ of $95 \%$ on the day of inoculation and ranging between 70 and $80 \%$ thereafter. All other experimental details related to the test for late blight resistance were identical to the laboratory assay described previously. The experiment comprised eight replications: the greenhouse was divided into eight blocks, each containing four plots that were randomly assigned to one of the four cultivars. No randomization of leaves was done for the resistance test, because leaves were not cut from the plants. The statistical analysis (REML) comprised the factors cultivar, leaf position, and their interaction as fixed, and the factors block, plot, and leaf as random.

\section{RESULTS}

The late blight resistance tests of all five experiments were completed before the final data analyses were performed. Data analyses were done separately, but in a similar way for each experiment. The linear lesion growth rates of $P$. infestans were used as input for the statistical analyses and resulted in estimations of averages for linear lesion growth rates and significance of the effects of the factors of interest (= the fixed part of the statistical analyses [REML]). These results are presented below.

Choice of leaf position over leaf age for the statistical analysis of the linear lesion growth rate of $P$. infestans. The factors leaf age and leaf position both were included in experiments 1, 3, and 4 . These factors were defined with approximately the same precision, obviously represented nearly the same source and amount of variation, and were highly intertwined. The comparison between the effects of leaf age and leaf position on the linear lesion growth rate of $P$. infestans is illustrated for cv. Alpha of experiment 3 . The same data were analyzed twice, once with leaf age and once with leaf position in the fixed part of the statistical analysis (REML). The first analysis showed that leaf age had a significant effect $(P<0.001)$ on the linear lesion growth rate of $P$. infestans. Generally, older leaves had higher linear lesion growth rates than younger leaves (Fig. 1A). In the same analysis, the effect of plant age on the linear lesion growth rate of $P$. infestans also was significant $(P<0.001)$. Older plants had lower linear lesion growth rates than younger plants (Fig. 1A). The interaction between leaf age and plant age was significant $(P<0.001)$, and the effects of both factors were of the same dimension. Therefore, the linear lesion growth rate of $P$. infestans of a specific leaf was determined equally by leaf age and plant age. The second analysis showed that leaf position had a significant effect $(P<0.001)$ on the linear lesion growth rate of $P$. infestans. Generally, basal leaves had higher linear lesion growth rates than top leaves (Fig. 1B). In this analysis, the effect of plant age on the linear lesion growth rate of $P$. infestans also was significant $(P<0.001)$. Usually, older plants had slightly lower linear lesion growth rates than younger plants (Fig. 1B). The interaction between leaf position and plant age was significant $(P=0.013)$, but the effects of leaf position were much larger than the effects of plant age. Therefore, the linear lesion growth rate of $P$. infestans of a specific leaf was determined predominantly by leaf position, whereas plant age had only a subordinate effect. As a result, presentation of the data is more clear and interpretation much easier when leaf position (Fig. 1B) is considered instead of leaf age (Fig. 1A).

In addition to these empirical results, there also was a theoretical ground for choosing leaf position instead of leaf age for the statistical analyses of the linear lesion growth rate of $P$. infestans. Successive analyses were performed to determine the relevance of leaf age and leaf position. The first statistical analyses (REML) were done with the factors cultivar, plant age, leaf age, and leaf position as fixed. The orders of leaf age and leaf position were exchanged in this analysis, and Wald statistics indicated that the contribution of leaf position was far more informative. In experiment 3, leaf position accounted for $70 \%$ of the total variation that was accounted for by the fixed part of the statistical analysis (REML), whereas leaf age accounted for only $59 \%$ of the total
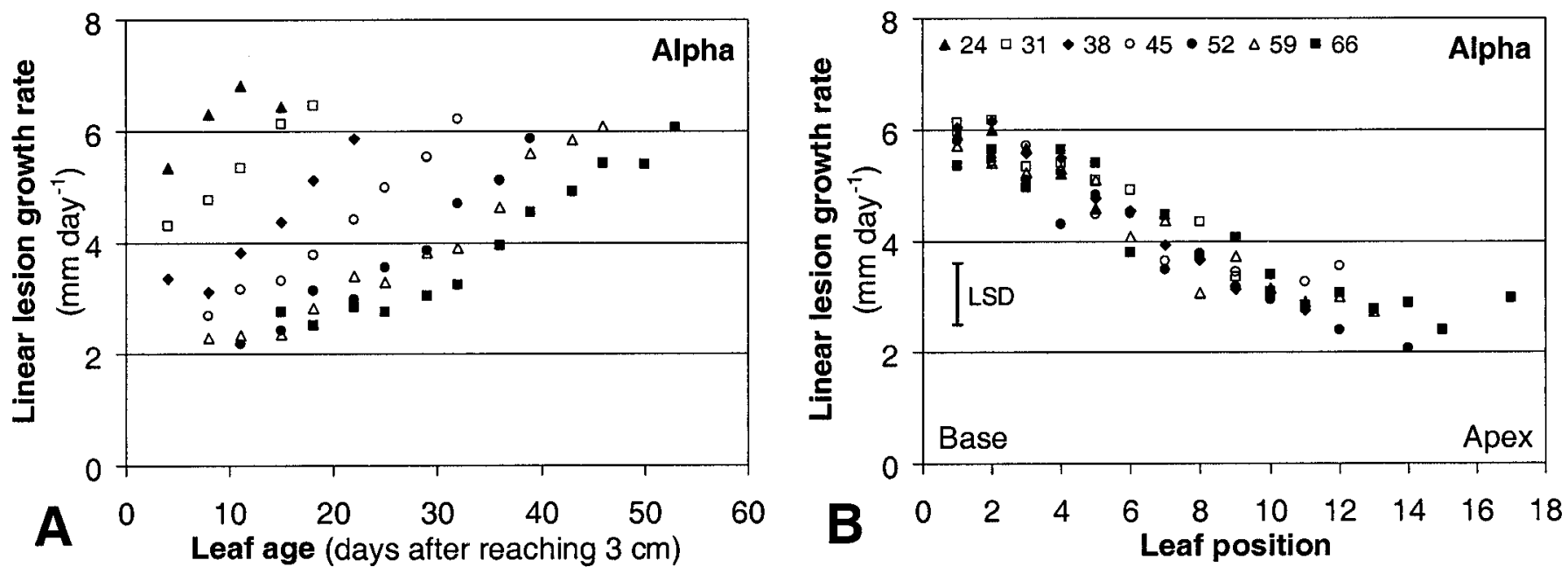

Fig. 1. Effect of $\mathbf{A}$, leaf age (least significant difference $\left.[\mathrm{LSD}]_{0.05}: 1.1\right)$ and of $\mathbf{B}$, leaf position $\left(\mathrm{LSD}_{0.05}: 1.1\right)$ on the linear lesion growth rate of Phytophthora infestans, given per plant age $(24,31,38,45,52,59$, or 66 days after planting). The same data were used for both graphs, but presented with different $x$ axes. Data from potato cv. Alpha of experiment 3 , which was performed in growth chambers. 
variation. Subsequent statistical analyses (REML) were done with the factors cultivar, plant age, and leaf age or leaf position as fixed. This analysis with leaf position accounted for much more variation in total (experiment 3: total Wald statistics 3,315) than the similar analysis with leaf age (experiment 3: total Wald statistics 2,373). Comparable results were found in all three experiments $(1,3$, and 4$)$, and both empirical and theoretical considerations resulted in choosing leaf position instead of leaf age for the final statistical analyses of the linear lesion growth rate of $P$. infestans for all experiments.

Experiments 1 and 2. Leaf position had a significant effect $(P<0.001)$ on the linear lesion growth rate of $P$. infestans. Generally, basal leaves had higher linear lesion growth rates than apical leaves (Figs. 2 and 3). This effect was similar for all plant ages (Fig. 2) and all cultivars (Figs. 2 and 3), except for cv. Eersteing, where basal and apical leaves had comparable linear lesion growth rates. The effect of plant age on the linear lesion growth rate of $P$. infestans also was significant $(P<0.001)$. Usually, older plants had slightly lower linear lesion growth rates than younger plants (Fig. 2). The interaction between plant age and leaf position was significant, but the effect was rather small (14\% of the total variation accounted for by the fixed part of the statistical analysis [REML]). In addition, the effect of cultivar on the linear lesion growth rate of $P$. infestans was significant $(P<0.001)$. Linear lesion growth rates were higher for Eersteling than for Pimpernel (Fig. 3; Table 1). The interaction between cultivar and leaf position was significant, but the effect small $(5 \%$ of the total variation accounted for by the fixed part of the statistical analysis [REML]).

Experiments 3 and 4. As in experiments 1 and 2, the effect of leaf position on the linear lesion growth rate of $P$. infestans was significant $(P<0.001)$. Basal leaves had, in general, higher linear lesion growth rates than apical leaves (Figs. 4 and 5), and this effect was similar for all plant ages and all cultivars (Figs. 4 and 5). In addition, plant age had a significant effect $(P<0.001)$ on the linear lesion growth rate of $P$. infestans. As in experiments 1 and 2, older plants usually had slightly lower linear lesion growth rates than younger plants (Figs. 4 and 5). The effect of cultivar on the linear lesion growth rate of $P$. infestans also was significant $(P<0.001)$. Linear lesion growth rates for Eersteling were higher than for Spunta and Alpha, and were lowest for Robijn (Figs. 4 and 5; Table 1). Some of the interactions between cultivar, plant age, and leaf position were significant, but their effects were always negligible (each less than $6 \%$ of the total variation accounted for by the fixed part of the statistical analysis [REML]).

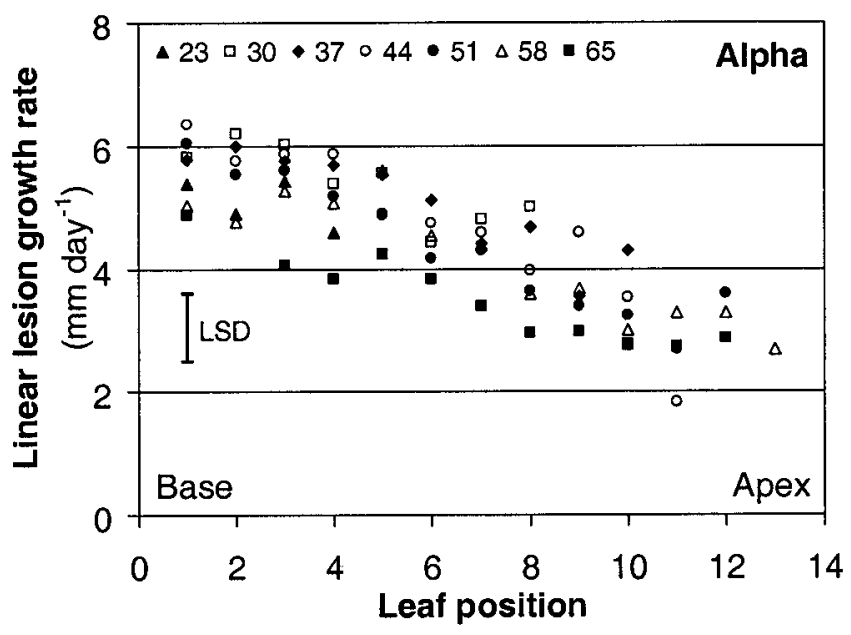

Fig. 2. Effect of leaf position on the linear lesion growth rate of Phytophthora infestans, given per plant age $(23,30,37,44,51,58$, or 65 days after planting). Data from experiment 1 , performed in a growth chamber with potato $\mathrm{cv}$. Alpha (least significant difference ${ }_{0.05}: 1.1$ ).
The effects of leaf position on the linear lesion growth rate of $P$. infestans were described by four-parameter logistic curves for experiment 3 (Table 2). Differences between cultivars only were significant $(P<0.001)$ for the lower asymptote (parameter A). The lower asymptote for Eersteling was higher than for Spunta and Alpha, and was lowest for Robijn (Table 2).

Experiment 5. In accordance with all previous experiments, leaf position had a significant effect $(P<0.001)$ on the linear lesion growth rate of $P$. infestans. Basal leaves generally had higher linear lesion growth rates than apical leaves (Fig. 6). This effect was similar for all cultivars (Fig. 6), except for cv. Eersteling, where the most basal leaves did not have the highest linear lesion growth rates. This dissimilarity of Eersteling also was found in experiment 2, and appeared to correlate with a high rate of senescence of the leaves after inoculation with $P$. infestans. The effect of cultivar on the linear lesion growth rate of $P$. infestans also was significant $(P<0.001)$. Linear lesion growth rates for Eersteling were higher than for Alpha, and were lowest for Robijn and Spunta (Fig. 6; Table 1). The interaction between cultivar and leaf position was significant, but the effect was rather small $(13 \%$ of the total variation accounted for by the fixed part of the statistical analysis [REML]).

\section{DISCUSSION}

The effects of plant age, leaf age, and leaf position on racenonspecific resistance against $P$. infestans were investigated in a series of field and controlled environment experiments with five different potato cultivars. The metric value for resistance was the linear lesion growth rate of $P$. infestans because this is the most representative component of resistance to late blight (6).

The first four experiments comprised seven late blight resistance tests performed on detached compound leaves in a controlled setting in the laboratory. All resistance tests resulted in the same relations between the studied factors (plant age, leaf age, and leaf position) and the linear lesion growth rate of $P$. infestans, and also in similar linear lesion growth rates for the different cultivars (Table 1); both are indicative of the repeatability of the resistance tests. Each of the first four experiments resulted in a ranking for the cultivars in agreement with data of long-term field evaluations for resistance to late blight (Table 1), indicating that the resistance tests also reflect the actual situation in the field. Similar relations were found between the studied factors (plant

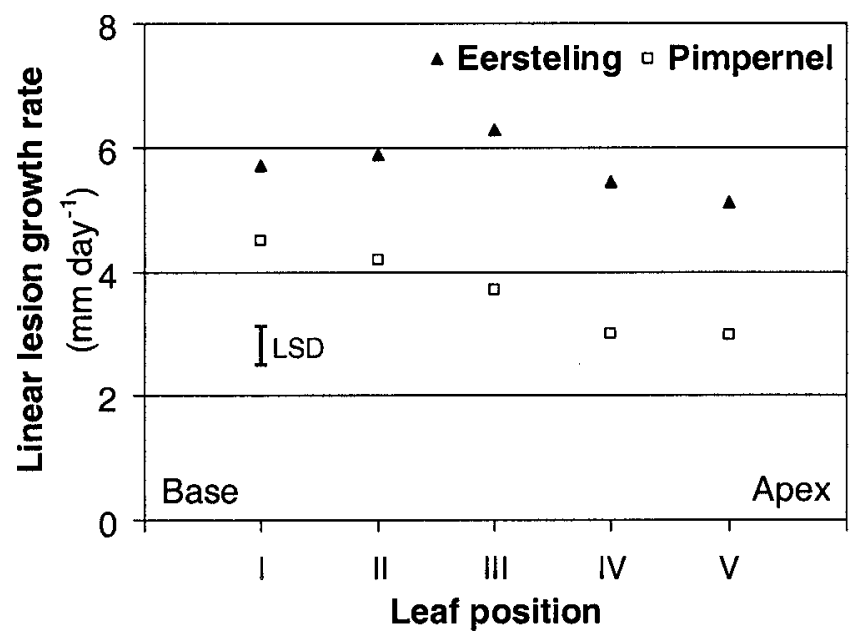

Fig. 3. Effect of relative leaf position on the linear lesion growth rate of Phytophthora infestans for plants $\approx 40$ days old (after emergence). The leaf positions were not exact, but five different leaves distributed evenly from base to apex were numbered $\mathrm{I}$ to $\mathrm{V}$, indicating their relative positions. Data from experiment 2 with potato cvs. Eersteling and Pimpernel that were grown in the field (least significant difference ${ }_{0.05}: 0.6$ ). 
age, leaf age, and leaf position) and the linear lesion growth rate of $P$. infestans in the fifth experiment, in which leaves were inoculated on the plants. The absolute levels of the linear lesion growth rate were somewhat lower in this resistance test, probably due to less favorable conditions for late blight development (lower RH) in the greenhouse, higher resistance to late blight of the upper leaf surface (33), or nondetachment of the leaves (19).

Leaf position proved to be the most significant of all considered factors and to have the largest effect on the linear lesion growth rate of $P$. infestans; apical leaves were far more resistant than basal leaves. Plant age and leaf age had only minor effects; thus, the resistance of a specific leaf remained about the same during its entire lifetime. This gradual increase in late blight resistance from basal leaves to apical leaves appeared to be a general effect, because it was found in all experiments, irrespective of cultivar, plant material (in vitro plantlets or seed tubers), growing conditions (climate-controlled or field), or resistance test (leaves detached or on the plant). The present study confirmed previous results that apical leaves were more resistant to late blight than basal leaves $(19,20)$.

Plant age also was a significant factor, but the effect on the linear lesion growth rate of $P$. infestans was much smaller than the effect of leaf position; in general, older plants were slightly more resistant than younger plants. Few previous studies found older plants to be more resistant to late blight than younger plants (5).
More frequent was the result that very young plants were susceptible, plants of intermediate age were the most resistant, and old plants (70 days after planting) $(11,27)$ became more susceptible again $(20,27)$. Most previous studies have focused on the effects of plant age and have not considered the effects of leaf position on resistance against $P$. infestans $(8,13,27)$. In the present study, the effects of leaf position amply exceeded the effects of plant age, and a large part of the effects of plant age on late blight resistance described previously probably can be attributed to leaf position. Each new leaf is slightly more resistant to late blight than the preceding one; thus, older plants with proportionally more of these more-resistant leaves will, on average, have a higher level of resistance for the entire plant.

Discrepancies with previous results also may have been caused by differences in experimental practices, to which differences in plant ages, growing conditions, and late blight resistance tests are the most likely contributors. It is hardly possible to estimate the effects of differences in resistance tests, because tests vary in type of material used for infection, quality of inoculum, method of inoculation, and evaluation of the symptoms. An apparent increase in susceptibility, in some cases, also can be caused by more favorable environmental conditions for late blight development during the resistance test (15). Actual plant ages in the present study were in accordance with the ones of previous research $(4,5,8,11,27)$, but differences in plant material (in vitro, tubers, or seeds) and differ-
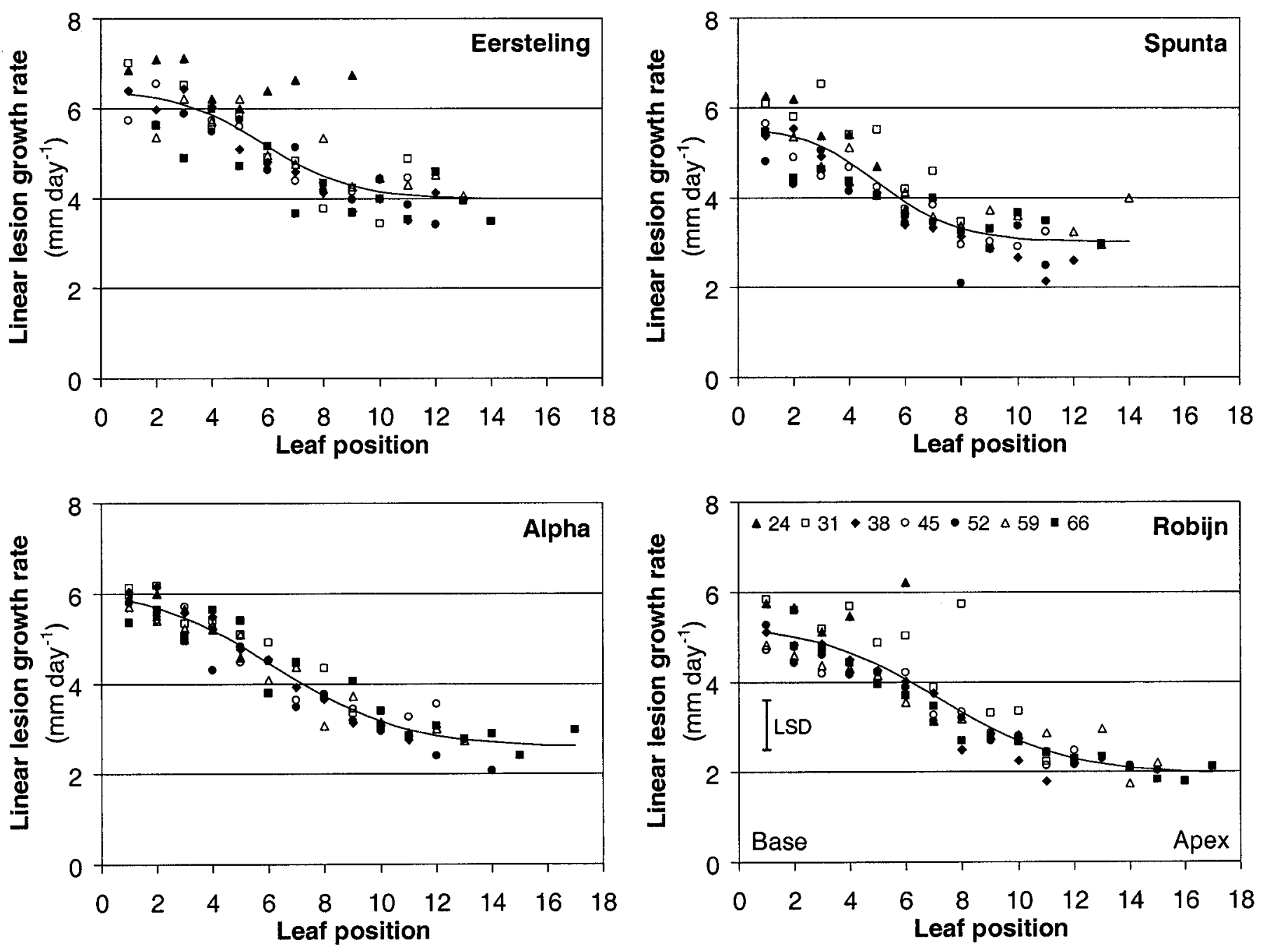

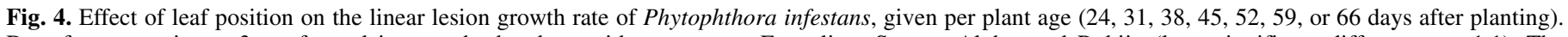

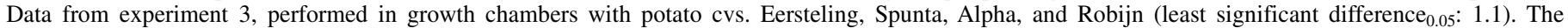
parameters of the logistic curves that were fitted in these graphs are given in Table 2. 
ences in growing conditions may have caused differences in physiological ages. Plants derived from in vitro material are known to develop more slowly than plants derived from seed tubers (18), and environmental factors also are known to influence plant development $(19,25)$. Moreover, environmental factors also affect late blight resistance $(24,32)$. In the present study, the influences of plant material or environmental conditions did not affect the relations found between the considered factors (plant age, leaf age, and leaf position) and the linear lesion growth rate of $P$. infestans. Still, increased susceptibility to late blight of plants at the final stage of plant development may have been undetected in the present study, because fully decayed leaves were not included in the experiments.

The factor leaf age in the present study corresponds to the apexto-base leaf positions used by Carnegie and Colhoun (4,5), where the youngest, most apical leaf was at position one. In both cases, the increase in the linear lesion growth rate of $P$. infestans was approximately linear from young to old leaves, with different levels of resistance for different plant ages (as in Figure 1A). The factor leaf position (counted from base to apex) represented nearly
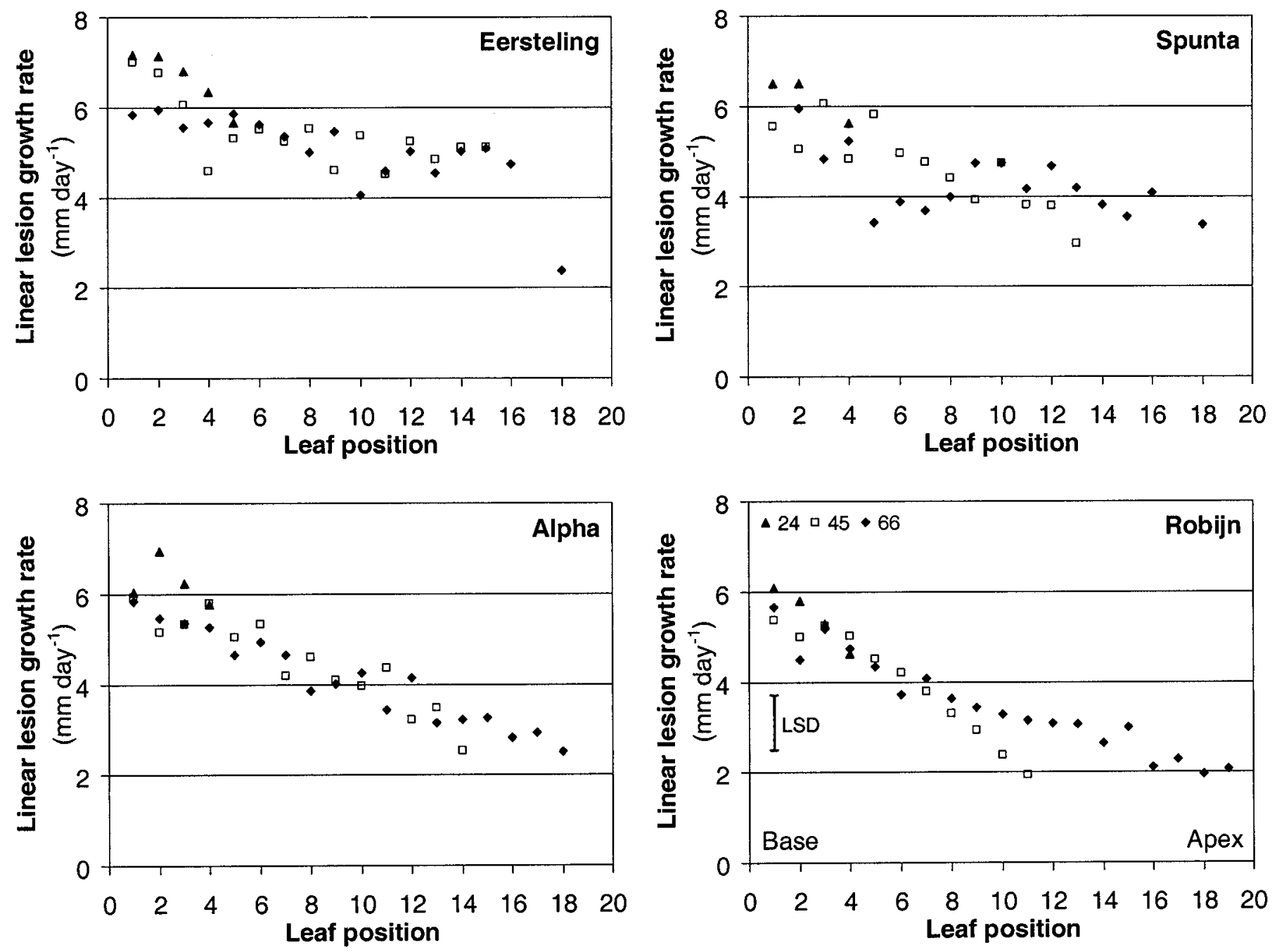

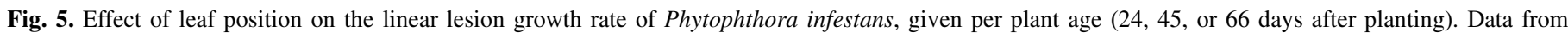
experiment 4 with potato cvs. Eersteling, Spunta, Alpha, and Robijn that were grown in the field (least significant difference ${ }_{0.05}$ : 1.2 ).

TABLE 2. Values for the parameters of the logistic curves fitted to the data of Figure 4, reflecting the effect of leaf position on the linear lesion growth rates of Phytophthora infestans of four potato cultivars grown in growth chambers

\begin{tabular}{|c|c|c|c|c|c|c|c|c|}
\hline \multirow[b]{2}{*}{ Cultivar } & \multirow[b]{2}{*}{ Foliage maturity type $^{\mathrm{v}}$} & \multirow[b]{2}{*}{ Late blight resistance ${ }^{\mathrm{w}}$} & \multirow[b]{2}{*}{$R^{2 \mathrm{x}}$} & \multicolumn{5}{|c|}{ Parameters logistic curves ${ }^{\mathrm{y}}$} \\
\hline & & & & $B$ & $M$ & $C$ & $A$ & $A+C$ \\
\hline Eersteling & 8.5 & 2 & 64 & -0.62 & 5.84 & 2.47 & $3.98 \mathrm{a}$ & 6.45 \\
\hline Spunta & 7 & 5 & 75 & -0.72 & 5.09 & 2.58 & $3.02 \mathrm{~b}$ & 5.60 \\
\hline Alpha & 4 & 5 & 92 & -0.43 & 6.18 & 3.62 & $2.58 \mathrm{bc}$ & 6.20 \\
\hline Robijn & 3 & 8 & 80 & -0.45 & 7.17 & 3.36 & $1.95 \mathrm{c}$ & 5.31 \\
\hline $\mathrm{LSD}_{0.05^{\mathrm{z}}}$ & $\ldots$ & $\ldots$ & $\ldots$ & 0.40 & 2.59 & 1.82 & 0.72 & 1.71 \\
\hline
\end{tabular}

${ }^{\mathrm{v}}$ Data of long-term field evaluations for foliage maturity type ( $1=$ late, $9=$ early), obtained from the Dutch list of varieties of field crops $(1-3)$.

${ }^{w}$ Data of long-term field evaluations for resistance to late blight $(1=$ susceptible, $9=$ resistant $)$, obtained from the Dutch list of varieties of field crops $(1-3)$.

x Adjusted $R^{2}(1-$ [mean squares regression]/[mean squares total] $)=$ percentage of variance accounted for.

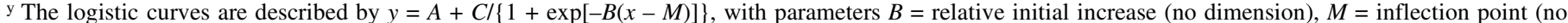
dimension), $C=$ increment $\left(\mathrm{mm} \mathrm{day}^{-1}\right), A=$ lower asymptote for $x=\infty\left(\mathrm{mm} \mathrm{day}^{-1}\right)$ and $A+C=$ upper asymptote for $x=0\left(\mathrm{~mm}\right.$ day $\left.{ }^{-1}\right)$. Different letters within a column indicate significant differences.

${ }^{\mathrm{z}} \mathrm{LSD}=$ least significant difference. 
the same source and amount of variation, but was, in comparison to the factor leaf age, hardly influenced by the factor plant age and, therefore, preferred in the statistical analyses.

The differences in the linear lesion growth rates of $P$. infestans between cultivars were significant and were in agreement with data of long-term field evaluations for resistance (Table 1). Only cv. Spunta in experiment 5 had relatively low linear lesion growth rates. The values for the lower asymptote (A) of the fitted curves of experiment 3 differed significantly between cultivars and showed correlation with the data of field evaluations for resistance to late blight, as well as for foliage maturity type (Table 2). The other factors tested (plant age, leaf age, and leaf position) and the other parameters of the logistic curves did not differ significantly between cultivars, indicating that these factors could not differentiate between early- and late-maturing cultivars or between resistant and susceptible ones. Such a differentiation would be useful to determine the crucial distinction between early- and late-maturing cultivars in relation to their late blight resistance. Despite the lack of significant differences between cultivars for most parameters of the logistic curves, the relative initial increase (B), inflection point (M), and increment (C) seemed to correlate with data of field evaluations for foliage maturity type, and the upper asymptote $(=\mathrm{A}+\mathrm{C})$ seemed to correlate with data of field evaluations for resistance to late blight (Table 2). These correlations are based on a set of only four cultivars; therefore, the results should be verified in an extended study including more cultivars that differ in foliage maturity type, resistance, or both.

Leaf position has major effects on late blight resistance; therefore, it is essential for a reliable comparison of different potato genotypes or treatments that leaves of the same position (counted from the base of the plant) are tested for resistance. Overlooking this consideration may result in ascribing contrasts in resistance against $P$. infestans to differences between genotypes or treatments, whereas they actually are caused by differences in leaf position. To avoid the effect of leaf position, resistance should be tested only on the more apical leaves, because the effect of leaf position is negligible in leaves of positions 10 and up.

It is commonly thought that basal leaves of potato plants in the field are earlier or more severely attacked by late blight (17) due to better microclimatic conditions for disease development in the lower parts of the crop (15). The present experiments with detached leaves prove that basal leaves are more susceptible to late blight than apical leaves, independent of plant architecture or environmental conditions. Therefore, a new strategy to control late

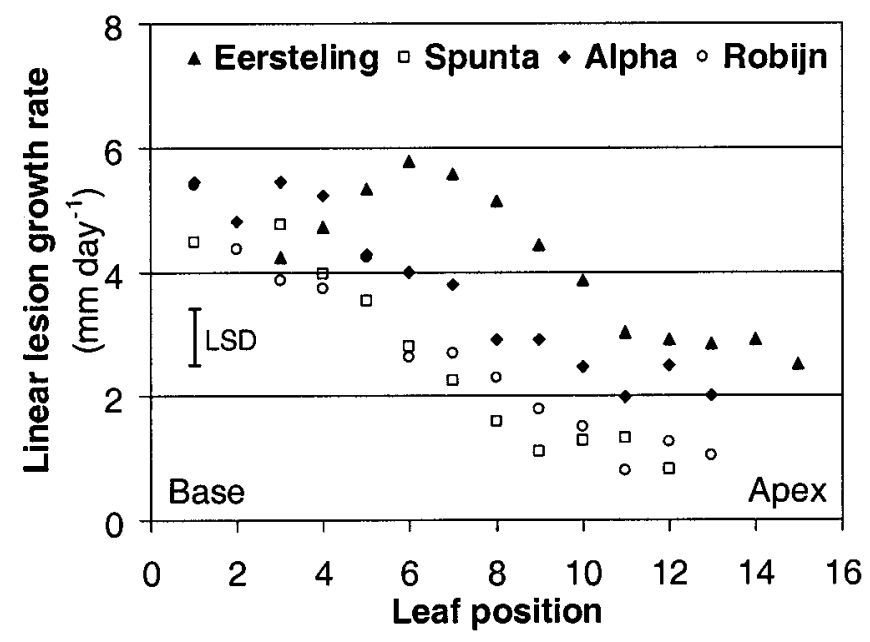

Fig. 6. Effect of leaf position on the linear lesion growth rate of Phytophthora infestans for plants of 35 days old (after planting). Data from experiment 5, performed in a climate-controlled greenhouse with intact plants of potato cvs. Eersteling, Spunta, Alpha, and Robijn (least significant difference $\left._{0.05}: 0.9\right)$. blight can be the breeding of cultivars that produce as many leaves as possible before the disease strikes.

\section{ACKNOWLEDGMENTS}

The research described in this article was financially supported by the Dutch Technology Foundation (STW; project WBI.3871). We thank L. Sijpkes for his contribution to the research; and E. van de Weg, R. Voorrips, T. den Nijs, and the members of the users' committee of project WBI.3871 of STW for suggestions on earlier drafts of the manuscript.

\section{LITERATURE CITED}

1. Anonymous. 1939. Pages 97-138 in: 15th List of varieties of field crops. Instituut Voor Plantenveredeling, Wageningen, The Netherlands.

2. Anonymous. 1988. Pages 196-257 in: 63rd List of varieties of field crops. Rijksinstituut voor het Rassenonderzoek van Cultuurgewassen (RIVRO), Wageningen, The Netherlands.

3. Anonymous. 2001. Pages 185-229 in: 76th List of varieties of field crops. Plant Research International, Wageningen, The Netherlands.

4. Carnegie, S. F., and Colhoun, J. 1980. Differential leaf susceptibility to Phytophthora infestans on potato plants of cv. King Edward. Phytopathol. Z. 98:108-117.

5. Carnegie, S. F., and Colhoun, J. 1982. Susceptibility of potato leaves to Phytophthora infestans in relation to plant age and leaf position. Phytopathol. Z. 104:157-167.

6. Colon, L. T., Budding, D. J., Keizer, L. C. P., and Pieters, M. M. J. 1995. Components of resistance to late blight (Phytophthora infestans) in eight South American Solanum species. Eur. J. Plant Pathol. 101:441-456.

7. Colon, L. T., Turkensteen, L. J., Prummel, W., Budding, D. J., and Hoogendoorn, J. 1995. Durable resistance to late blight (Phytophthora infestans) in old potato cultivars. Eur. J. Plant Pathol. 101:387-397.

8. Dorrance, A. E., and Inglis, D. A. 1997. Assessment of greenhouse and laboratory screening methods for evaluating potato foliage for resistance to late blight. Plant Dis. 81:1206-1213.

9. Flier, W. G. 2001. Variation in Phytophthora infestans, sources and implications. Ph.D. thesis. Wageningen University, The Netherlands.

10. Forbes, G. A., Hijmans, R. J., and Nelson, R. J. 1998. Potato blight: A world problem. Paper number 5.1.9S in: Abstracts of the 7th International Congress of Plant Pathology. British Society for Plant Pathology, Edinburgh, UK.

11. Fry, W. E., and Apple, A. E. 1986. Disease management implications of age-related changes in susceptibility of potato foliage to Phytophthora infestans. Am. Potato J. 63:47-56.

12. Gebhardt, C., and Valkonen, J. P. T. 2001. Organization of genes controlling disease resistance in the potato genome. Annu. Rev. Phytopathol. 39:79-102.

13. Grainger, J. 1956. Host nutrition and attack by fungal parasites. Phytopathology 46:445-456.

14. Grünwald, N. J., Cadena Hinojosa, M. A., Rubio Covarrubias, O., Rivera Peña, A., Niederhauser, J. S., and Fry, W. E. 2002. Potato cultivars from the Mexican national program: Sources and durability of resistance against late blight. Phytopathology 92:688-693.

15. Harrison, J. G. 1992. Effects of the aerial environment on late blight of potato foliage, a review. Plant Pathol. 41:384-416.

16. Hodgson, W. A. 1961. Laboratory testing of the potato for partial resistance to Phytophthora infestans. Am. Potato J. 38:259-264.

17. Lapwood, D. H. 1961. Potato haulm resistance to Phytophthora infestans. III. Lesion distribution and leaf destruction. Ann. Appl. Biol. 49:704-716.

18. Lommen, W. J. M. 1999. Causes for low tuber yields of transplants from in vitro potato plantlets of early cultivars after field planting. J. Agric. Sci. 133:275-284.

19. Lowings, P. H., and Acha, I. G. 1959. Some factors affecting growth of Phytophthora infestans (Mont.) De Bary. I. P. infestans on living potato leaves. Trans. Br. Mycol. Soc. 42:491-501.

20. Mooi, J. C. 1965. Experiments on testing field resistance to Phytophthora infestans by inoculating cut leaves of potato varieties. Eur. Potato J. 8:182-183.

21. Murashige, T., and Skoog, F. 1962. A revised medium for rapid growth and bio assays with tobacco tissue cultures. Physiol. Plant 15:473-497.

22. Ordoñez, M. E., Forbes, G. A., and Trognitz, B. 1998. Relationship between ineffective $R$-genes and expansion rate of lesions on potato leaves, caused by Phytophthora infestans. Plant Pathol. 47:130-136.

23. Patterson, H. D., and Thompson, R. 1971. Recovery of inter-block information when block sizes are unequal. Biometrika 58:545-554.

24. Phukan, S. N. 1993. Effect of plant nutrition on the incidence of late blight disease of potato in relation to plant age and leaf position. Indian 
J. Mycol. Plant Pathol. 23:287-290.

25. Pohjakallio, O., Salonen, A., and Antila, S. 1957. Analysis of earliness in the potato. Acta Agric. Scand. 7:361-388.

26. Ross, H. 1986. Late blight, Phytophthora infestans (Mont.) de Bary. Pages 82-86 in: Advances in Plant Breeding, vol. 13. Potato Breeding: Problems and Perspectives. H. Ross, ed. Verlag Paul Parey, Berlin.

27. Stewart, H. E. 1990. Effect of plant age and inoculum concentration on expression of major gene resistance to Phytophthora infestans in detached potato leaflets. Mycol. Res. 94:823-826.

28. Stewart, H. E., Flavelle, P. H., McCalmont, D. C., and Wastie, R. L. 1983. Correlation between glasshouse and field tests for resistance to foliage blight caused by Phytophthora infestans. Potato Res. 26:41-48.

29. Stewart, H. E., Taylor, K., and Wastie, R. L. 1983. Resistance to late blight in foliage (Phytophthora infestans) of potatoes assessed as true seedlings and as adult plants in the glasshouse. Potato Res. 26:363-366.

30. Swiezynski, K. M. 1990. Resistance to Phytophthora infestans in potato cultivars and its relation to maturity. Genet. Pol. 31:99-106.

31. Toxopeus, H. J. 1958. Some notes on the relations between field resistance to Phytophthora infestans in leaves and tubers and ripening time in
Solanum tuberosum subsp. tuberosum. Euphytica 7:123-130.

32. Umaerus, V. 1959. The relationship between peroxidase activity in potato leaves and resistance to Phytophthora infestans. Am. Potato J. 36:124-131.

33. Umaerus, V. 1969. Studies on field resistance to Phytophthora infestans. 1. The infection efficiency of zoospores of $P$. infestans as influenced by the host genotype. Z. Pflanzenzüchtg. 61:29-45.

34. Vleeshouwers, V. G. A. A., Van Dooijeweert, W., Govers, F., Kamoun, S., and Colon, L. T. 2000. The hypersensitive response is associated with host and nonhost resistance to Phytophthora infestans. Planta 210:853864.

35. Vleeshouwers, V. G. A. A., Van Dooijeweert, W., Keizer, L. C. P., Sijpkes, L., Govers, F., and Colon, L. T. 1999. A laboratory assay for Phytophthora infestans resistance in various Solanum species reflects the field situation. Eur. J. Plant Pathol. 105:241-250.

36. Wastie, R. L. 1991. Breeding for resistance. Pages 193-224 in: Advances in Plant Pathology, vol. 7, Phytophthora infestans: The Cause of Late Blight of Potato. D. S. Ingram and P. H. Williams, eds. Academic Press, London. 